ORIGINAL ARTICLE

\title{
Capillary refill: prognostic value in Kenyan children
}

\author{
A Pamba, K Maitland
}

Arch Dis Child 2004;89:950-955. doi: 10.1136/adc.2003.032516

\begin{abstract}
Aims: To determine whether delayed capillary refill time ( $>3$ seconds) is a useful prognostic indicator in Kenyan children admitted to hospital.

Methods: A total of 4160 children admitted to Kilifi District Hospital with malaria, malarial anaemia, acute respiratory tract infection (ARI), severe anaemia (haemoglobin $<50 \mathrm{~g} / \mathrm{l}$ ), gastroenteritis, malnutrition, meningitis, or septicaemia were studied.

Results: Overall, delayed capillary refill time (dCRT), present in $346 / 4160(8 \%)$ of the children, was significantly more common in fatal cases $(44 / 189,23 \%)$ than survivors $(7.5 \%)$, and had useful prognostic value. In children admitted with malaria, gastroenteritis, or malnutrition, likelihood ratio tests suggested that dCRT was useful in identifying high risk groups for mortality, but its prognostic value in anaemia, ARI, and sepsis was unclear due to low case fatality or limited numbers. The severity features of impaired consciousness and deep breathing were significantly associated both with the presence of dCRT and fatal outcome. In children, with either of these severity features, a less stringent value of $d C R T(>2 s)$ identified $50 \%$ of children with hypotension (systolic BP $<2 \mathrm{SD}$ ) and $40 \%$ of those requiring volume resuscitation (for metabolic acidosis).

Conclusions: Although CRT is a simple bedside test, which may be used in resource poor settings as a guide to the circulatory status, dCRT should not be relied on in the absence of other features of severity. In non-severe disease, the additional presence of hypoxia, a moderately raised creatinine (>80 $\mu \mathrm{mol} / \mathrm{I})$, or a raised white cell count should prompt the need for fluid expansion.
\end{abstract}

See end of article for authors' affiliations

Correspondence to: Dr K Maitland, KEMRI/ Wellcome Trust Unit, PO Box 230, Kilifi, Kenya; kmaitland@kilifi.mimcom. net

Accepted 25 January 2004
$T$ he context of emergency medical care in sub-Saharan Africa (SSA) is very different from that provided by modern casualty departments in the developed world. In Africa, the burden on the outpatient and emergency services is much greater and resources are limited to simple low technology assessments, which are readily available and inexpensive. Thus, in most circumstances the assessment of circulation in a sick child presenting to hospital for treatment is limited to simple clinical assessments. Capillary refill time (CRT) is one simple bedside tool that may be useful in the assessment of circulation in a sick child presenting to hospital for treatment. The assessment of CRT has become a standard part of the rapid, structured approach to the initial assessment and to prioritise initial management. ${ }^{12}$ However, opinion on its value as a clinical sign of poor perfusion remains divided, since it has been shown to vary with the presence of oedema, characteristics of a patient's skin, ambient temperature, and the amount of pressure applied. ${ }^{3-7}$ In adults, delayed CRT (dCRT) has no proven diagnostic value. ${ }^{8}$ Nevertheless dCRT has been included in a prognostic scoring system for use in African meningococcal epidemics, ${ }^{9}$ and more recently has been incorporated in the World Health Organisation (WHO) emergency triage assessment and treatment (ETAT) guidelines that were developed for use in developing countries. ${ }^{10}$ These guidelines define shock on the basis of dCRT ( $>3$ seconds), cold hands, and a weak fast pulse, and recommend volume expansion in such children. Validation of the ETAT guidelines in children presenting to casualty in Brazil and Malawi however, showed that shock was diagnosed in remarkably few children $(<0.5 \%)$, and case fatality in those diagnosed as being in shock was $~ 100 \% .{ }^{11}{ }^{12}$ This raises questions about the validity of these signs to detect children in whom simple forms of resuscitation may be life saving. Furthermore, these studies also bring into question the reproducibility of these signs between different observers. In a separate paper, we examine inter-observer variation in the detection of clinical signs used to define shock. ${ }^{12 a}$ In this study we examine the utility of dCRT ( $>3 \mathrm{~s}$ ) in determining prognosis in acute admissions to a paediatric ward in a tropical setting, which to our knowledge has not been previously evaluated.

\section{PATIENTS AND METHODS \\ Study site and population}

The study was conducted at Kilifi District Hospital (KDH) on the coast of Kenya. Since 1989 a programme of clinical research has been conducted at the hospital by a team from the Kenya Medical Research Institute (KEMRI). The team is responsible for the paediatric inpatient services, which include a seven bed high dependency unit (HDU) for the management and treatment of children with severe, life threatening disease. Approximately 5000 children are admitted each year. Owing to limited bed space, the two major indications for transfer to the HDU are impaired consciousness (prostration or coma) and deep "acidotic" breathing, which define the main high risk groups requiring urgent intervention. Detailed descriptions of the inpatient population and denominator population of the hospital have been published previously. ${ }^{13}$ In this study, we retrospectively examined admission data from every child admitted to the paediatric ward and HDU with one of eight diagnostic syndromes during the period between l September 1999 and 31 December 2000. Young infants, under 3 months of age, were however excluded from the analysis.

Abbreviations: ARI, acute respiratory tract infection; $\mathrm{BP}$, blood pressure; CRT, capillary refill time; dCRT, delayed capillary refill time; (>3 seconds); CVP, central venous pressure; GE, gastroenteritis; $\mathrm{Hb}$, haemoglobin; HDU, high dependency unit; KDH, Kilifi District Hospital; LR, likelihood ratio; MA, malaria anaemia; NCHS, National Child Health Surveillance; SD, standard deviation; SS, severity sign; WBC, white cell count; WHO, World Health Organisation 


\section{Clinical assessment}

Medically qualified members of the KEMRI team assess all children admitted to the ward. A standard examination and admission proforma are completed and treatment is generally prescribed per protocol. Details of the standard clinical assessment have been reported elsewhere. ${ }^{15}{ }^{16}$ CRT was assessed by applying pressure on a finger for 3 seconds, then counting the time required for the blanched digit to fully reperfuse. Coma was assessed according to the Blantyre Coma Score. ${ }^{17}$ Temperature gradient and pulse volume were only recorded from September 2000, and hence data is limited to 1058 cases. Temperature gradient was assessed by running the back of the palm of the hand down the lower limb and radial pulse was used to assess pulse volume. The vast majority of acute medical admissions were rationalised into eight major final diagnostic categories. These diagnoses were made at discharge and were based on the major clinical features and interventions received. These are summarised, together with their definitions in table 1. Since malaria parasitaemia was common, the diagnosis of malaria did not solely depend on the presence of parasitaemia, but was made by exclusion of other major syndromes.

\section{Data analysis}

Within each major syndrome group, the prevalence of dCRT in fatal cases and survivors was compared using $\chi^{2}$ tests or Fisher's exact tests. Likelihood ratios (LR) and 95\% confidence intervals (CI) were calculated to express the likelihood of dCRT being present in fatal cases compared to survivors. Dichotomous categorical variables were constructed for the following triage severity signs (SS): extreme tachycardia ( $>180$ beats/minute in children $\leqslant 1$ year or $>160$ in those $>1$ year); hypoxia (oxygen saturation $<90 \%$ by pulse oximeter); tachypnoea (respiratory rate $>60$ breaths/minute); and hypothermia (axillary temperature $<36^{\circ} \mathrm{C}$ ). The definitions for the derived variables were included in the Pediatric Advanced Life Support (PALS) guidelines indicating a definitive need for urgent therapeutic intervention. ${ }^{2}$ Conscious level was grouped into four categories: normal, lethargic, prostrate (unable to sit unsupported or breast feed), and coma (inability to localise pain). Likelihood ratios $(95 \%$ CI $)$ were used to examine the likelihood of a SS predicting death separately and when the SS was observed in combination with dCRT. Multivariate logistic regression (MLR) models were used to identify risk factors associated with death among all admissions, and to identify variables that were associated with dCRT. Results are reported as odds ratios (ORs) and 95\% confidence intervals. MLR models initially included all the categorical triage variables (see above), fever (axillary temperature $>37.5^{\circ} \mathrm{C}$ ), patient derived data (age, sex, anthropometry), peripheral malaria parasitaemia, bacteraemia (blood culture proven), and derived categorical variables ( $>95 \%$ confidence intervals for normality) for routinely collected laboratory data (hypoglycaemia $<2.5 \mathrm{mmol} / \mathrm{l}$ and creatinine $>80 \mu \mathrm{mol} / \mathrm{l}$ ) and continuous variables for haemoglobin and white cell count. $\mathrm{Z}$ scores for the anthropometric parameter weight-for-age (WA), were calculated for each individual using Epi Info v 2000 (CDC, Atlanta) which compares data to the National Center for Health Statistics (NCHS) reference values. Variables were sequentially dropped from the model if the association with the dependent variable was non-significant $(p \geqslant 0.1)$. The final model included only the variables that were significantly associated with the outcome variable $(\mathrm{p}<0.05)$. Temperature gradient and pulse volume were excluded from these models due to limited data. MLR models also examined variables associated with fatal outcome in the subgroup of children transferred to the HDU.

\section{RESULTS}

Between 1 September 1999 and 31 December 2000, 6259 children were admitted to $\mathrm{KDH}$. Of these, 875 were $<3$ months old and were excluded from the analysis. A total of 4344 children were admitted with one of the eight primary diagnoses, in whom CRT was assessed in 4160. Of these, 1994 $(48 \%)$ were girls and 2166 (52\%) were boys. The median age at admission was 1.6 years (interquartile range 0.8-3.0). A total of 616 children (14.6\%) were transferred to the high dependency unit for urgent treatment, and 189/4160 children died (crude fatality rate was $4.5 \%$ ). Table 2 shows the numbers of patients admitted by diagnosis. Secondary diagnoses for each category are summarised beneath the table. Malaria parasitaemia was not solely confined to children diagnosed with malaria. The proportion of children infected with Plasmodium falciparum malaria differed between clinical syndrome groups, being present in 120/373 (32\%) with severe malnutrition, 15/58 (26\%) with meningitis, 141/ $620(23 \%)$ with GE, and 104/862 (12\%) with ARI. All children with positive malaria slides received standard first line treatment for malaria regardless of primary diagnosis. Admission blood cultures were positive in $\leqslant 2 \%$ of children admitted with a primary diagnosis of malaria, MA, GE, or anaemia. Bacteraemia was more common in children admitted with a primary diagnosis of ARI $(6 \%)$, malnutrition (14\%), meningitis $(41 \%)$, or sepsis $(67 \%)$. The organisms most commonly cultured were Streptococcus pneumoniae, group A streptococci, and Haemophilus influenzae. Eight per cent of malnourished children grew a Gram negative organism. Neisseria meningitidis was not isolated during this period (in blood cultures or CSF culture).

\section{Prevalence and prognostic value of delayed capillary refill}

Overall, 3272/4160 (79\%) children had a CRT of $\leqslant 2$ s, 542 (13\%) had a CRT of $2-3$ s, and $346(8 \%)$ had a CRT of $>3$ s. Mortality in each of these CRT categories was 103/3256 (3\%),

Table 1 Definition of the major diagnostic syndrome categories

\begin{tabular}{ll}
\hline Disease syndrome & Definition \\
\hline Malaria & Fever $\left(>37.5^{\circ} \mathrm{C}\right.$ ) plus asexual stage malaria parasites in the absence of another major syndrome \\
Malaria anaemia (MA) & As above, plus severe anaemia (haemoglobin $<50 \mathrm{~g} / \mathrm{l})$ \\
Acute respiratory tract & Respiratory rate greater than 90 th centile for age and the presence of cough or respiratory tract signs \\
infection (ARI) & Weight for height $<70 \% \mathrm{NCHS} / \mathrm{WHO}$ reference value, ${ }^{15}$ or visible severe wasting (marasmus), or bilateral symmetrical pedal \\
Malnutrition (PCM) & oedema (oedematous malnutrition) \\
Gastroenteritis (GE) & Three or more vomits and $/$ or 3 or more loose stools in the preceding 24 hours \\
Anaemia & Haemoglobin less than $50 \mathrm{~g} / /$ (without malaria parasites) \\
Meningitis & Abnormal lumbar puncture findings consistent with infection ${ }^{13}$ \\
Sepsis & Fever $\left(>37.5^{\circ} \mathrm{C}\right.$ ) in the absence of malaria parasites and focal signs \\
\hline
\end{tabular}


Table 2 Prevalence and prognostic ability of delayed capillary refill in common childhood illnesses

\begin{tabular}{|c|c|c|c|c|c|c|c|}
\hline $\begin{array}{l}\text { Admission } \\
\text { syndrome }\end{array}$ & Cases, $\mathrm{n}$ & CRT >3 s (\%) & $\begin{array}{l}\text { Survivors with dCRT/ } \\
\text { total survivors (\%) }\end{array}$ & $\begin{array}{l}\text { Deaths with dCRT/ } \\
\text { total deaths (\%) }\end{array}$ & $\chi^{2}$ & $\mathbf{p}$ & $\begin{array}{l}\text { Likelihood ratio* } \\
(95 \% \mathrm{Cl})\end{array}$ \\
\hline Malaria & 1654 & $102(6)$ & $90 / 1609(6)$ & $12 / 45(27)$ & 34 & $<0.0001$ & 4.8 (2.6 to 8.7$)$ \\
\hline$M A$ & 424 & 70 (17) & $68 / 418(16)$ & $2 / 6(33)$ & $1.2 \dagger$ & 0.26 & $2.0(0.5$ to 8.3$)$ \\
\hline ARI & 862 & 31 (4) & $30 / 843$ (4) & $1 / 19(5)$ & $0.12 \dagger$ & 0.5 & $1.5(0.2$ to 12.9$)$ \\
\hline Malnutrition & 373 & $53(14)$ & $34 / 292(12)$ & $19 / 81(24)$ & 7.3 & 0.007 & 2.0 (1.2 to 3.5$)$ \\
\hline Gastroenteritis & 620 & $47(8)$ & $43 / 602$ (7) & $4 / 18(22)$ & $5.7 \dagger$ & 0.04 & 3.1 (1.1 to 8.7 ) \\
\hline Anaemia & 147 & 39 (27) & $35 / 141(25)$ & $4 / 6(67)$ & 5.2 & 0.04 & $2.7(1$ to 7.5$)$ \\
\hline Meningitis & 59 & $0(0)$ & $0 / 53(0)$ & $0 / 6(0)$ & - & - & - \\
\hline Sepsis & 21 & 4 (19) & $2 / 13(15)$ & $2 / 8(25)$ & $0.3 \dagger$ & 0.6 & $1.63(0.23$ to 11.6$)$ \\
\hline Total & 4160 & 346 (8) & $302 / 3971$ (8) & $44 / 189$ (23) & 58 & $<0.0001$ & 3.06 ( 2.24 to 4.21$)$ \\
\hline
\end{tabular}

Secondary diagnoses: malaria (34 GE, 64 ARI, 26 malnutrition); ARI (23 GE, 22 anaemia 19); malnutrition (GE 26, ARI 16, anaemia 19); and gastroenteritis (anaemia 6, ARI 22).

†Fisher's exact test.

$38 / 538(7 \%)$, and 44/341 (13\%) respectively $\left(\chi^{2}=79.0, \mathrm{df}=2\right.$, $\mathrm{p}<0.0001)$. Numerically, the greatest number of cases with dCRT was among children admitted with a primary diagnosis of malaria (102/1653; 6\%); however, dCRT was more prevalent among children admitted with severe anaemia $(27 \%)$, sepsis $(19 \%)$, MA $(17 \%)$, or malnutrition $(14 \%)$ (table 2). In general, the likelihood ratios suggest that the use of dCRT has some value as a diagnostic test intending to identify a high risk group ( $\mathrm{LR}=3.06,95 \%$ CI 2.2 to 4.2 ) (table 2). The prognostic utility of dCRT was greatest among cases of malaria, gastroenteritis, and malnutrition. However, the confidence intervals for both malnutrition and gastroenteritis suggest that the utility of this sign to prognosticate in these conditions remains uncertain. Similarly, in children with non-malarial anaemia, dCRT had some value in distinguishing those at risk of death; however, the confidence interval spans from differences, which would have little clinical relevance. Although dCRT was not significantly associated with death for children with MA, ARI, and sepsis, confidence intervals were wide and clinically important associations could not be discounted. For these groups in particular, larger sample sizes are needed to determine prognostic significance of dCRT.

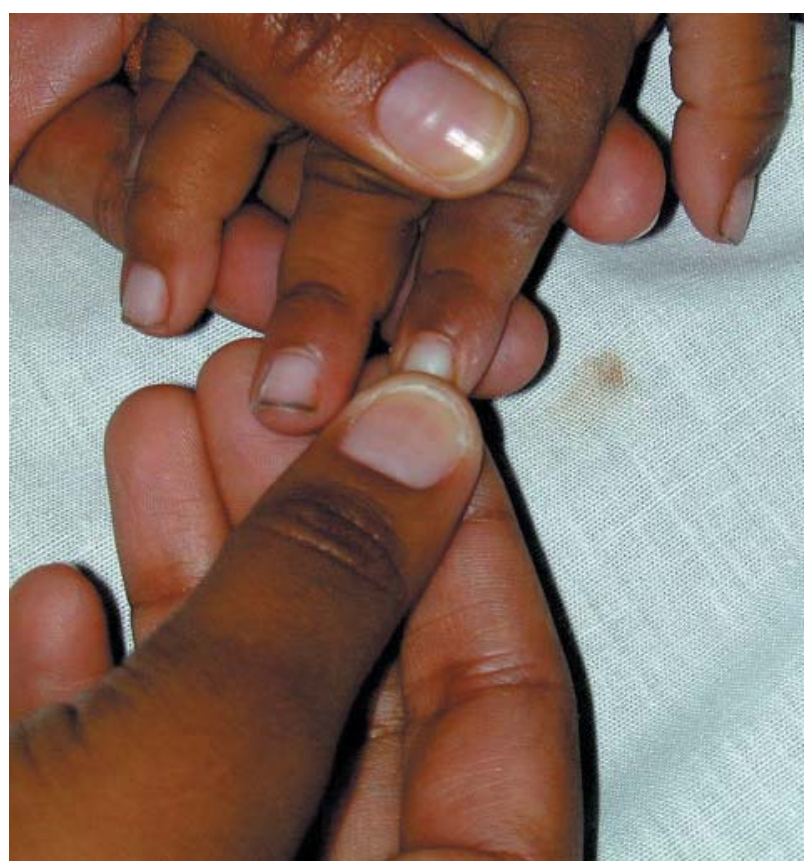

Figure 1 Capillary refill testing: a valuable triage sign?

\section{Capillary refill time in conjunction with other signs of severity}

A total of $93 / 3597$ (2.3\%) children who were admitted to the general paediatric ward died. Although a statistically greater number of those dying had a dCRT, 19 (20\%) compared to $227(6.5 \%)$ survivors $\left(\chi^{2}=28, p<0.001\right)$, capillary refill time was neither sensitive nor specific enough to adequately identify a well defined group at high risk of dying. For this reason we examined how dCRT performed when combined with other triage features of severity in all study patients. Table 3 describes the prevalence of dCRT in children with SSs indicative of cardiorespiratory and/or neurological compromise $^{12}$ and the likelihood ratio of death solely attributable to the SS and when the SS was associated with dCRT. Severe tachycardia (heart rate $>160$ beats/min) was common (48\%) in all admissions but present in only $3 \%$ of deaths, thus had poor prognostic value. Similarly, the presence of tachypnoea (respiratory rate $>60$ breaths $/ \mathrm{min}$ ) was not associated with any prognostic significance. Hypoxia, deep breathing, weak pulse volume, and impaired consciousness (prostration or coma) were less frequently diagnosed at admission $(\leqslant 8 \%)$ but were present in $25 \%, 28 \%, 18 \%, 14 \%$, and $23 \%$ of deaths respectively. The likelihood ratios suggest that any one of these severity signs is very good at identifying high risk groups. Dehydration was common in admissions $(12 \%)$ and present in $42 \%$ of deaths. Likelihood ratios indicate that the presence of dehydration has significant prognostic implications. However, this should be interpreted with caution since $25 \%(129 / 373)$ of the children with malnutrition were assessed as clinically dehydrated, and mortality was significantly greater in this group 46/129 (36\%) compared to 38/ $224(14 \%)$ of malnourished children without signs of dehydration $\left(\chi^{2}=22, \mathrm{df}=1, \mathrm{p}<0.001\right)$. In other words these deaths represented $46 / 79(58 \%)$ of the deaths in children who were deemed "clinically dehydrated".

LRs were computed to examine the likelihood of a child with dCRT and an SS being a fatal case or a survivor. In children with hypothermia, weak pulse volume, hypoxia, indrawing, deep breathing, and impaired conscious level, the likelihood of fatal outcome was significantly greater than survival in the presence dCRT (see table 3 ).

\section{Variables associated with a fatal outcome and dCRT}

Table 4 summarises the variables that were significantly associated with a fatal outcome and dCRT on multivariate analysis. Any impairment of consciousness, anthropometric indices of severe malnutrition, deep breathing, and hypoxia were all significantly associated with a fatal outcome. dCRT was, however not independently associated with death. Of the laboratory investigations, a moderately raised creatinine $(>80 \mu \mathrm{mol} / \mathrm{l})$, a bacteraemia, and a raised white cell count (WBC) were associated with an increased risk of death. 


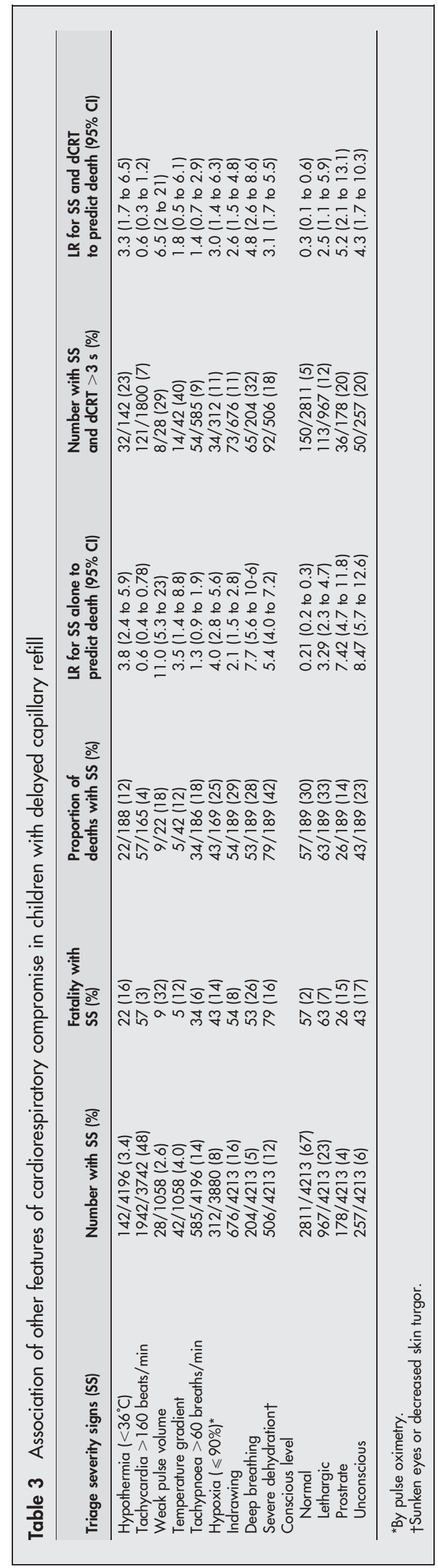

For dCRT the only significant associations were impairment of consciousness, deep breathing, hypothermia, and clinical features of dehydration. Haemoglobin concentration and fever were negatively associated with death. Noteworthy is the lack of association between capillary refill time and hypoxia (defined by pulse oximetry) and serum creatinine, which were independently associated with a fatal outcome. Factors such as age, heart rate, respiratory rate, indrawing, and acidosis (base excess) were not independently associated with fatal outcome or dCRT. However, acidosis (base excess) was partially predicted by deep breathing.

\section{CRT in severe illness}

Children with impaired consciousness or deep "acidotic" breathing were the major indications for direct admission to the HDU. Delayed CRT was more common in these children, being present in 103/616 (17\%) compared to $246 / 3597$ (7\%) in children admitted to the general paediatric ward $\left(\chi^{2}=68\right.$, $\mathrm{p}<0.0001)$. Ninety six $(15.5 \%)$ children admitted to the HDU died. Of the children who died, 25 (26\%) had a dCRT, compared to $78 / 520(15 \%)$ in survivors $\left(\chi^{2}=7.1, p=0.008\right)$. Of the deaths that occurred within 24 hours of admission to the HDU, 12/27 (44\%) had a dCRT, which contrasted with dCRT being more prevalent, 6/21 (29\%), in the later deaths (day 1-2) in children admitted to the general ward.

Blood pressure was measured on admission to the HDU in 603 children; of these, 96 (16\%) had hypotension, and in this group $25(26 \%)$ were assessed as having a dCRT. Intravenous boluses of normal saline ( $10-50 \mathrm{ml} / \mathrm{kg}$ ) were administered in $267(43 \%)$ children, principally to treat acidosis (base excess $<-8$ ) or shock (blood pressure $<-2$ SD mean). Delayed CRT was present in $63(24 \%)$ of these children. Using a less stringent definition of dCRT ${ }^{1}$ we found a delayed CRT $>2 \mathrm{~s}$ was present in 49/96 (51\%) hypotensive children and 106/267 $(40 \%)$ children requiring volume expansion. On multiple logistic regression analysis, four factors were associated with a fatal outcome in children transferred to the HDU: hypoxia (oxygen saturation $<90 \%$ on pulse oximetry) (OR 2.5; 95\% CI 1.4 to $4.4 ; \mathrm{p}=0.002) ; \mathrm{a}$ moderately raised creatinine $(>80 \mu \mathrm{mol} / \mathrm{l}) \quad(\mathrm{OR} \quad 2.4 ; 95 \%$ CI 1.2 to $4.2 ; \mathrm{p}=0.002)$; hypotension (OR $2.3 ; 95 \%$ CI 1.3 to $4.2 ; \mathrm{p}=0.006$ ); and deep breathing (OR 2.2; 95\% CI 1.2 to 3.7; p < 0.0001). Malaria parasitaemia was associated with a reduced risk of death (OR $0.3 ; 95 \%$ CI 0.18 to $0.5 ; \mathrm{p}<0.001$ ).

\section{DISCUSSION}

We have shown that dCRT ( $>3 \mathrm{~s}$ ) is common $(8 \%)$ in Kenyan children admitted to hospital and that mortality was higher in children who had a dCRT at admission, whether they were subsequently managed on the general ward $(20 \%$ in deaths versus $6 \%$ in survivors) or were transferred to the HDU $(25 \%$ in deaths versus $15 \%$ in survivors). In children admitted with the primary diagnoses of malaria, gastroenteritis, malnutrition, and malarial anaemia the likelihood ratios for a fatal outcome were $\geqslant 2.0$, implying that a dCRT was a reasonable bedside prognostic indicator. However, owing to the range of the confidence intervals for gastroenteritis, malnutrition, and malarial anaemia the potential use of this clinical sign in predicting a fatal outcome in these conditions is uncertain. In ARI and non-malarial anaemia clinically important associations between dCRT and outcome could not be assessed adequately due to the limited numbers examined.

In children admitted to the general paediatric ward (with non-severe disease), mortality was higher in those with a dCRT $(6 \%)$ than in those without $(2 \%)$, suggesting that the significance of dCRT in this group was not sufficiently weighted in the clinical assessment. However, the potential risk of recommending an increase in the number of intravenous fluid prescriptions in a relatively poorly 
Table 4 Variables associated with a fatal outcome and delayed capillary refill time on multivariate analysis

\begin{tabular}{|c|c|c|c|c|}
\hline & \multicolumn{2}{|l|}{ Fatal outcome } & \multicolumn{2}{|l|}{ Delayed capillary refill } \\
\hline & Odds ratio $(95 \% \mathrm{Cl})$ & $\mathbf{p}$ & Odds ratio $(95 \% \mathrm{Cl})$ & 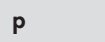 \\
\hline Unconscious & 4.1 (2.1 to 7.8$)$ & $<0.0001$ & $2.2(1.5$ to 3.4$)$ & $<0.0001$ \\
\hline Prostrate & $3.9(1.9$ to 7.6$)$ & $<0.0001$ & $2.2(1.2$ to 2.1$)$ & $<0.0001$ \\
\hline Lethargy & $2.2(1.3$ to 3.6$)$ & 0.002 & $1.6(1.2$ to 2.1$)$ & 0.001 \\
\hline Deep breathing & 3.1 (1.8 to 5.2$)$ & $<0.0001$ & $2.6(1.8$ to 3.6$)$ & $<0.0001$ \\
\hline Hypoxia ( $\leqslant 90 \%$ ) & $3.1(1.9$ to 5.0$)$ & $<0.0001$ & - & \\
\hline Fever $\left(>37.5^{\circ} \mathrm{C}\right)$ & - & & $0.5(0.4$ to 0.7$)$ & $<0.0001$ \\
\hline Hypothermia $\left(<36^{\circ} \mathrm{C}\right)$ & - & & $2.3(1.4$ to 3.7$)$ & $<0.0001$ \\
\hline Severe dehydration & - & & $3.2(2.4$ to 4.4$)$ & $<0.0001$ \\
\hline WAZ $<-3$ & 5.1 (3.4 to 7.6$)$ & $<0.0001$ & - & \\
\hline Malaria parasitaemia & $0.36(0.23$ to 0.5$)$ & $<0.0001$ & - & \\
\hline Haemoglobin & - & & 0.75 (0.72 to 0.79$)$ & $<0.0001$ \\
\hline Creatinine $>80 \mu \mathrm{mol} / \mathrm{l}$ & $2.2(1.3$ to 3.6$)$ & $<0.0001$ & - & \\
\hline Bacteraemia & $2.1(1.5$ to 2.9$)$ & $<0.0001$ & - & \\
\hline White cell count & $1.02(1.0$ to 1.03$)$ & 0.003 & - & \\
\hline
\end{tabular}

monitored group of children may outweigh the benefit. Moreover, most of the deaths, $66 / 93(71 \%)$ in this patient group, occurred after 48 hours, which also questions the utility of dCRT in this setting as the sole guide to fluid management. We suggest that dCRT should, therefore, be interpreted in conjunction with other features of hypovolaemia, such as those suggested as part of the SIRS model and employed in this study (hypoxia, a moderately raised creatinine, or raised WBC $)^{18}$ or those suggested by others. ${ }^{19} 20$ Unfortunately, the general application of this guideline is restricted by limited availability of pulse oximetry and creatinine measurement at most hospitals in SSA.

In a previous study at Kilifi, Berkley and colleagues showed that immediate mortality (within four hours of admission) was significantly higher in children admitted with impaired consciousness (prostration or coma), deep breathing, severe anaemia, or hypothermia. ${ }^{13}$ One explanation for the rapid demise within this group, is that these features indicated the presence of un-resuscitated shock. Capillary refill time or other features of shock, however, were not part of the routine clinical assessment at that time. In the current study we found that $44 \%$ of deaths occurring $<24$ hours after admission to the HDU had a dCRT, suggesting that this sign has some value in prioritising management. In these children a less stringent value of CRT $>2$ s identified $>50 \%$ of those with hypotension and $40 \%$ of children requiring urgent volume expansion. With regard to deep breathing, we have previously shown that metabolic acidosis is present in over $95 \%$ of children with malaria complicated by this clinical feature. ${ }^{21}$ As a result, at $\mathrm{KDH}$ we routinely manage children with deep breathing with either volume resuscitation or an urgent transfusion (if $\mathrm{Hb}<50 \mathrm{~g} / \mathrm{l}$ ). Furthermore, observational and intervention studies have now established that hypovolaemia is common in children with severe malaria complicated by acidosis; both acidosis and hypovolaemia resolved safely with volume expansion. ${ }^{22-24}$

Children with impaired consciousness have traditionally been relatively volume restricted due to fears of inducing or worsening raised intracranial pressure in a number of conditions of infectious aetiology. Recent studies suggest that this approach may be deleterious in a number of conditions..$^{26}$ Decreased consciousness and seizures are frequent complications of circulatory impairment, the latter being often overlooked as a central cause is more frequently assumed. The definition of sepsis and related syndromes (now know as the systemic inflammatory response syndrome, SIRS) has recently been reviewed. ${ }^{18}$ Apart from the cardiovascular signs of impaired tissue perfusion, features of major organ dysfunction such as hypoxia, reduced urine output, agitation, decreased consciousness and seizures, and metabolic derangement (including hypoglycaemia and electrolyte imbalance) are included in the definition. We have shown that any decrease in conscious level is one of the principal prognostic indicators. Owing to the limitations of a retrospective study to adequately address such issues, we are unable to provide new evidence in support of either approach to management. Nevertheless, our results do suggest that shock may complicate a proportion of the cases with impaired consciousness with dCRT, and that fluid resuscitation may be beneficial in such cases. Before future recommendations can be made, further studies are required to investigate the indications, safety, and efficacy of fluid resuscitation in such children.

Finally, in the group of children with severe malnutrition, dCRT showed a reasonable correlation with fatal outcome; both of these features were also present in $>50 \%$ of these deaths, which were complicated by the clinical features of severe dehydration. There are at least two reasons why the results of this retrospective analysis should be interpreted with caution. First, the assessment of hydration status in a child with severe malnutrition is complicated, since sunken eyes and decreased elasticity are both features of chronic, severe malnutrition. ${ }^{27}$ Second, current guidelines for the management of severe malnutrition recommend the avoidance of intravenous fluids in the absence of decompensated shock, ${ }^{28}$ due to concerns about circulatory overload and pulmonary oedema, since the cardiovascular changes in this condition are thought to represent an adaptive hypocirculatory state. ${ }^{29}{ }^{30}$ We recommend that, in the absence of studies examining the role of hypovolaemia in severe malnutrition, the current WHO guidelines still apply when managing children with severe malnutrition.

In conclusion, we have shown that dCRT is a reasonable prognostic indicator, particularly in children with malaria. In children with life threatening disease (impaired consciousness and/or deep breathing), a less stringent definition of $\mathrm{dCRT}>2 \mathrm{~s}$ is an adequate indicator of the need for volume expansion; however, a bolus of fluid should be cautiously administered to any child with impaired consciousness. In children with non-severe disease, $\mathrm{dCRT}>3 \mathrm{~s}$ should not be used as a sole guide for volume replacement, but should be interpreted in combination with other features, particularly those that predict a poor outcome. The significance of dCRT in children with severe malnutrition cannot be interpreted in the absence of contemporary information regarding the haemodynamic status of this group. In order to establish better guidelines, prospective studies should try to define an objective definition of shock, including other easily detectable 
bedside markers of impaired perfusion (for example, temperature gradient and pulse volume), particularly in children with impaired consciousness.

\section{ACKNOWLEDGEMENTS}

The authors would like to thank the medical and nursing staff of Kilifi District Hospital and KEMRI/Wellcome Trust, Kilifi; Amanda Ross for statistical advice; Ian Machoniche for reading an early version of the paper; and Tom Williams for editing the final version. $\mathrm{K}$ Maitland is supported by the Wellcome Trust, UK. This manuscript is published with the permission of the Director of KEMRI.

This paper is dedicated to the late Maxwell Ochieng, whose great courage was inspirational.

\section{Authors' affiliations}

A Pamba, Centre for Geographic Medicine Research, Coast, KEMRI/

Wellcome Trust Unit, PO Box 230, Kilifi, Kenya

K Maitland, Department of Academic Paediatrics, Imperial College, London, UK

\section{REFERENCES}

1 Advanced paediatric life support: the practical approach, 1 st edn. London: BMJ Publishing Group, 1993.

2 American Heart Association. Pediatric Advanced Life Support 1997-1999. Emergency cardiovascular care programs. American Heart Association, 1997.

3 Saveeda J, Harris G, Li S. Capillary refilling in the assessment of dehydration. Am J Dis Child 1991;145:296-8.

4 Schriger D, Baraff L. Capillary refill: is it a useful predictor of hypovolaemic states? Ann Emerg Med 1991; 20:601-5.

5 Gorelick M, Shaw K, Baker M. Effects of ambient temperature on capillary refill in healthy children. Pediatrics 1993:92:699-702.

6 Tibby S, Hatherill M, Mardoch I. Capillary refill and core-peripheral temperature gap as indicators of haemodynamic status in paediatric intensive care patients. Arch Dis Child 1999:60:163-6.

7 Bumke K, Maconochie I. Paediatric capillary refill times. Trauma 2001;3:217-20

8 McGee S, Abernethy Wr, Simel D. The rational clinical examination. Is this patient hypovolemic? JAMA 1999;17:1022-9.

9 Ajayi-Obe E, Lodi E, Alkali A, et al. Prognostic scores for use in African meningococcal epidemics. Bull World Health Organ 1998;76:149-52.

10 Gove S, Tamburlini G, Molyneux E, et al. Development and technical basis of simplified guidelines for emergency triage assessment and treatment in developing countries. WHO Integrated Management of Childhood Illness (IMCI) Referral Care Project. Arch Dis Child 1999;81:473-7.
11 Tamburlini G, Di Mario S, Maggi R, et al. Evaluation of guidelines for emergency triage assessment and treatment in developing countries. Arch Dis Child 1999;81:478-82.

12 Robertson MMEM. Triage in the developing world-can it be done? Arch Dis Child $2001 ; 85: 208-13$

12a Otieno H, Were E, Ahmed I, et al. Are bedside features of shock reproducible between different observers? Arch Dis Child 2004;89:977-9.

13 Berkley JA, Ross A, Mwangi I, et al. Prognostic indicators of early and late death in children admitted to district hospital in Kenya: cohort study. BMJ 2002;326:361-7.

14 Snow RW, Bastos de Azevedo I, Lowe BS, et al. Severe childhood malaria in two areas of markedly different falciparum transmission in east Africa. Acta Trop 1994; 57:289-300.

15 Berkley J, Mwarumba S, Bramham K, et al. Bacteraemia complicating severe malaria in children. Trans R Soc Trop Med Hyg 1999:93:283-6.

16 English M, Punt J, Mwangi I, et al. Clinical overlap between malaria and severe pneumonia in Africa children in hospital. Trans $R$ Soc Trop Med Hyg 1996;90:658-62

17 Molyneux ME, Taylor TE, Wirima JJ, et al. Clinical features and prognostic indicators in paediatric cerebral malaria: a study of 131 comatose Malawian children. Q J Med 1989;71:441-59.

18 Saez-Llorens X, Vargas S, Guerra F, et al. Application of new sepsis definitions to evaluate outcome of pediatric patients with severe systemic infections. Pediatr Infect Dis J 1995;14:557-61.

19 Gorelick M, Shaw K, Murphy K. Validity and reliability of clinical signs in the diagnosis of dehydration in children. Pediatrics 1997;99:E6

20 Vega RMAJ. A prospective study of the usefulness of clinical and laboratory parameters for predicting percentage of dehydration in children. Pediatr Emerg Care 1997;13:179-82.

21 English M, Waruiru C, Amukoye E, et al. Deep breathing in children with severe malaria: indicator of metabolic acidosis and poor outcome. Am J Trop Med Hyg 1996;55:521-4.

22 English $M$, Saverwein R, Waruiru $C$, et al. Acidosis in severe childhood malaria. Q J Med 1997;90:263-70.

23 Maitland K, Levin M, English $M$, et al. Severe P. falciparum malaria in Kenyan children: evidence for hypovolaemia. Q J Med 2003;96:427-34.

24 Maitland K, Pamba A, Newton C, et al. Response to volume resuscitation in children with severe malaria. Pediatr Crit Care Med 2003:4:426-31.

25 Powell KR, Sugarman LI, Eskenazi AE, et al. Normalization of plasma arginine vasopressin concentrations when children with meningitis are given maintenance plus replacement fluid therapy. J Pediatr 1990; 1 17:515-22.

26 Herson VC, Todd JK. Prediction of morbidity in Hemophilus influenzae meningitis. Pediatrics 1977:59:35-9.

27 WHO. Management of the child with a serious infection or severe malnutrition. Geneva: World Health Organisation, 2000.

28 WHO. Management of the child with a serious infection or severe malnutrition. Geneva: World Health Organisation, 2000:89-90.

29 Alleyne G. Cardiac function in severely malnourished children. Clin Sci 1967;30:533-62

30 Kothari SSP, Shetalwad TM, Patel AN, et al. Left ventricular mass and function in children with severe protein energy malnutrition. Int J Cardiol 1992;35:19-25. 